\title{
Structure secondaire et contrôle de l'épissage alternatif
}

L'importance de l'épissage alternatif comme mécanisme de contrôle de l'expression des gènes n'est plus à démontrer. Les nombreux travaux parus dans la littérature montrent que l'épissage alternatif joue un rôle prédominant dans la programmation de processus biologiques variés [1]. A titre d'exemple, on peut citer le déterminisme sexuel chez la drosophile, la production de protéines possédant un pouvoir anti-oncogénique, la mise en place de programmes morphogéniques, ou encore la synthèse de protéines dépendant de la différenciation tissulaire et/ou du stade de développement.

Un des problèmes fascinants soulevés par l'épissage alternatif, a trait au mécanisme de sélection qui s'exerce sur certains sites d'épissage et qui conduit à l'incorporation ou à l'exclusion d'un exon particulier dans l'ARN messager. Le plus souvent, les sites d'épissage bordant ces exons montrent peu de variation par rapport aux séquences consensus et ne peuvent expliquer, à eux seuls, la reconnaissance sélective de ces exons. Il est évident que des changements intervenant dans les dinucléotides invariants $\mathrm{GT}$ et $\mathrm{AG}$ (respectivement sites 5' et 3' d'épissage) tels qu'on peut les observer dans certaines affections aboutissent aussi à l'exclusion d'un exon mais ne peuvent être assimilés à un cas classique d'épissage alternatif. L'analyse d'un nombre croissant de gènes indique que le contrôle de l'épissage alternatif est dû à l'action conjointe d'éléments actifs en cis présents sur le transcrit primaire et de facteurs agissant en trans. Si l'identification des séquences en cis s'est accrue au cours de ces dernières années, la nature des facteurs agissant en trans reste le plus souvent à élucider. S'agit-il de facteurs généraux d'épissage ou de facteurs spécifiques? Pour les premiers, des études récentes suggèrent que des modifications dans la concentration de certains facteurs ubiquitaires pourraient intervenir. Pour les seconds, les seuls exemples connus concernent la cascade des gènes intervenant dans le déterminisme sexuel chez la drosophile où, grâce à l'apport de la génétique, certains régulateurs ont pu être identifiés. Quant au mécanisme moléculaire à l'origine de l'épissage alternatif, il demeure dans la plupart des cas inconnu.

Depuis cinq ans, nous nous intéressons à la régulation de l'expression de deux exons mutuellement exclusifs (6A et $6 \mathrm{~B})$, présents dans la partie interne du gène de la $\beta$-tropomyosine de poulet [2]. Le choix de l'un ou l'autre de ces deux exons dépend de la différentiation musculaire : l'exon $6 \mathrm{~A}$ est présent dans les ARN messagers des tissus non musculaires et des muscles lisses, l'exon 6B est présent uniquement dans ceux des muscles striés (figure 1). In vitro, dans des extraits nucléaires de cellules HeLa (qui expriment la forme non musculaire de la $\beta$-tropomyosine), un précurseur s'étendant de l'exon $6 \mathrm{~A}$ à l'exon 7 donne le messager attendu : $6 \mathrm{~A}$ fusionné à 7 avec exclusion de l'exon spécifique du muscle strié, $6 \mathrm{~B}$. Nous avons montré que l'inhibition de l'épissage de l'intron (IVS AB) entre les deux exons mutuellement exclusifs est due en partie à la présence d'une longue suite de pyrimidines qui sépare le point de branche-

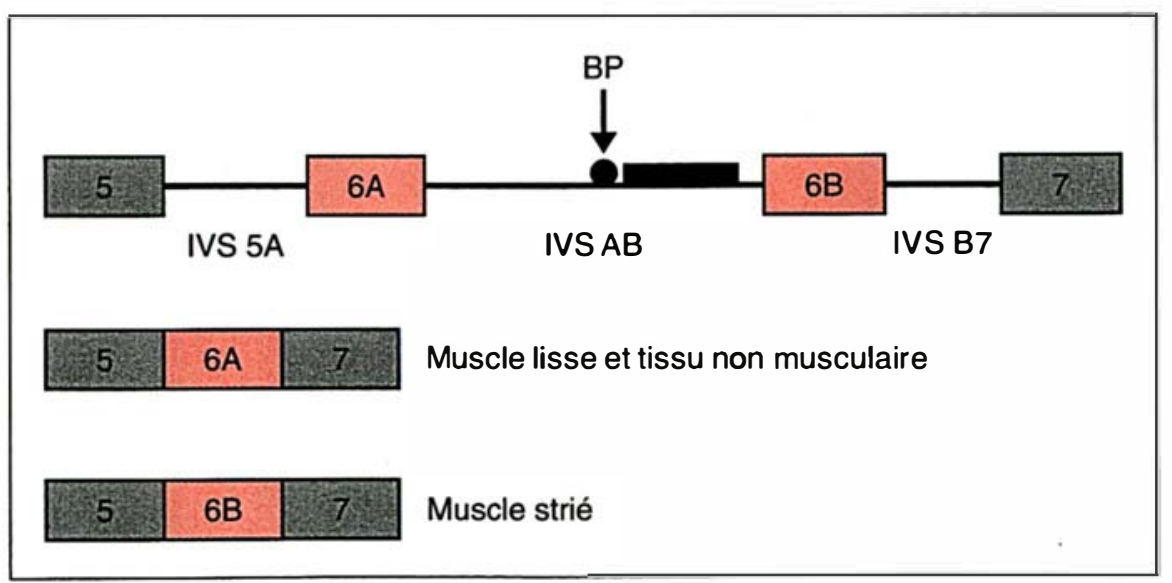

Figure 1. Organisation du gène de la $\beta$-tropomyosine dans sa partie interne. Dans les cellules non musculaires et les muscles lisses, l'épissage donne un ARNm 5-6A-7 avec exclusion de l'exon spécifique du muscle strié 6B. A l'inverse, dans les muscles striés et les myotubes, l'exon $6 A$ est exclu et l'ARNm contient les exons 5-6B-7. BP indique le point de branchement de I'intron IVS AB situé à 105 nt du site 3' d'épissage précédant l'exon 6B. Le rectangle noir représente la séquence riche en pyrimidines longue de 96 nucléotides. 
ment et le site 3' d'épissage de l'intron précédent l'exon $6 \mathrm{~B}$. La délétion de cette séquence ou sa substitution par une séquence "neutre" entraîne une stimulation importante de l'épissage de l'intron IVS AB. Le plus remarquable est que ces mêmes mutations provoquent également une forte stimulation de l'épissage de l'intron en aval de l'exon 6B, IVS B7 [3]. La répression de l'épissage de l'intron IVS B7 ne peut être attribuée à une simple compétition entre deux sites 5' d'épissage (celui en aval de $6 \mathrm{~A}$ étant plus fort que celui en aval de 6B) vis-à-vis du site 3' d'épissage précédent l'exon 7. En effet, l'élimination de l'exon 6A ne suffit pas pour lever l'inhibition [4]. En revanche, l'élimination de l'exon $6 \mathrm{~A}$ et de la suite de pyrimidines stimule l'épissage de l'intron IVS B7 [4]. L'ensemble de ces données nous a suggéré que la répression de l'épissage de l'intron IVS B7 pouvait être due à la formation d'une structure secondaire autour de l'exon $6 \mathrm{~B}$, empêchant sa reconnaissance dans les cellules non musculaires. Cette hypothèse, déjà soulevée en 1989 par Libri et al. [5], a été confirmée par deux approches complémentaires.
Dans la première approche, nous avons déterminé la structure secondaire dans la région de l'exon squelettique, à l'aide de sondes chimiques et enzymatiques [6]. Comme le montre le modèle de la figure $2 A$, l'exon $6 \mathrm{~B}$ est engagé dans plusieurs structures en tige et boucle, et maintenu dans une structure fermée par une longue tige (III) formée de l'appariement de la portion 3' terminale de l'intron IVS $A B$, riche en pyrimidines, avec la partie proximale de l'intron IVS B7. Pour prouver la validité de ce modèle, nous avons introduit deux types de mutations :

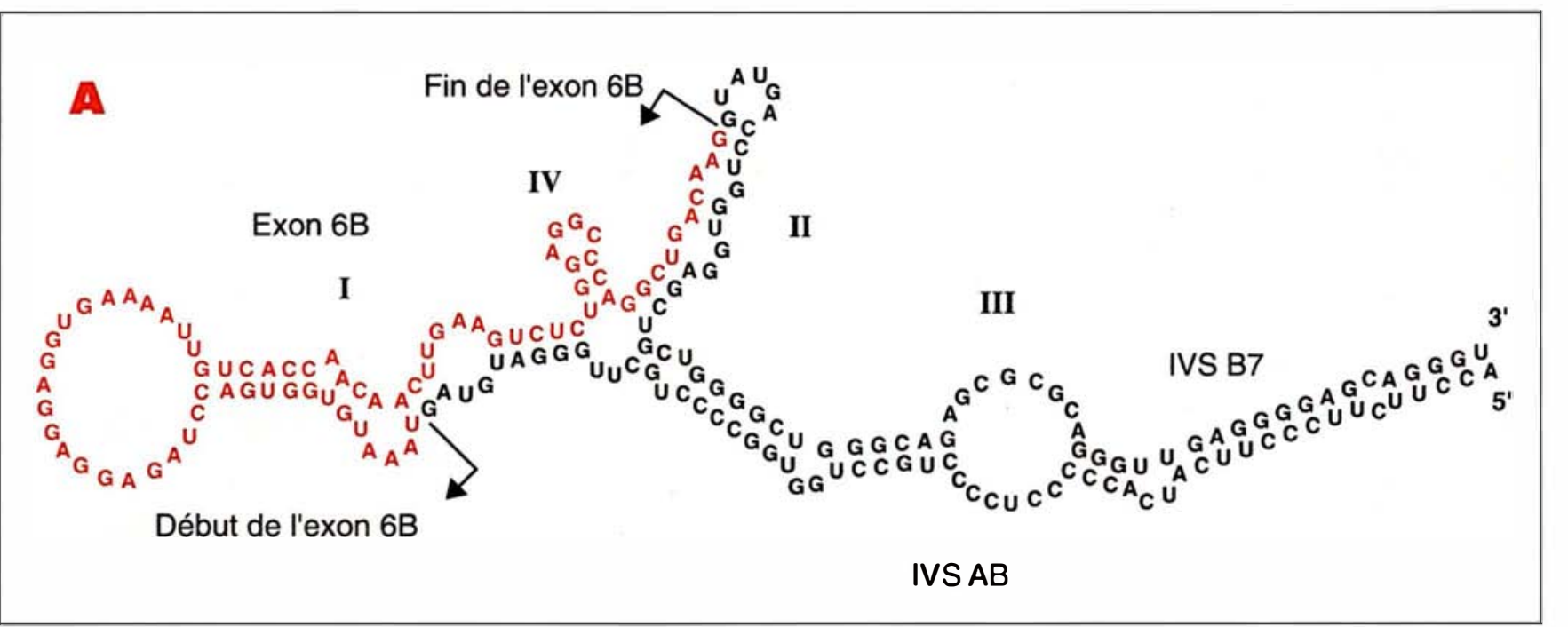

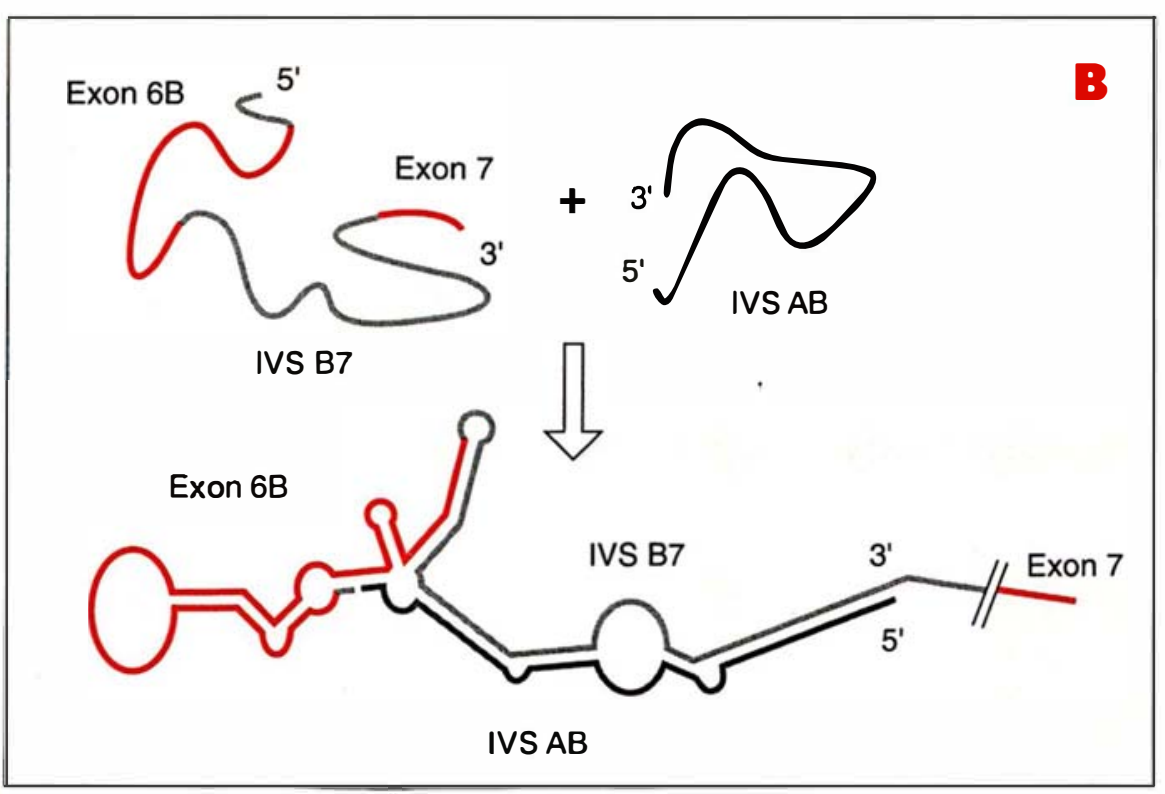

954
Figure 2. A. Structure secondaire de I'ARN autour de I'exon 6B. La structure secondaire a été déterminée en analysant les interactions ARNARN à l'aide de nucléases spécifiques des régions en simple brin et double brin et $d^{\prime}$ agents chimiques de modifications de bases. Le modèle thérique a été choisi pour satisfaire à la fois la cartographie expérimentale et les contraintes physico-chimiques calculées par ordinateur. La structure montre les 70 derniers nucléotides de l'intron IVS $A B$ avec la séquence riche en pyrimidines, l'exon squelettique $6 B$ len rougel et les 60 nucléotides de la partie proximale de l'intron IVS B7. Les flèches indiquent les jonctions exon/intron. Les nombres I, II, III, IV indiquent les structures en tige et en boucle. B. Représentation schématique de la reconstitution de la structure. 
des mutations simples sur les brins ascendants et descendants, destinées à déstabiliser les structures en hélice, et des doubles mutations compensatoires qui, bien que modifiant la structure primaire, sont destinées à restaurer la complémentarité de bases. Comme attendu, les mutations simples entraînent une dérépression de l'épissage de l'intron IVS B7, alors que les mutations compensatoires répriment de nouveau l'épissage de l'intron. In vivo, Libri et al. ont montré que la formation de la tigeboucle I est aussi nécessaire pour empêcher la reconnaissance de l'exon 6B dans les myoblastes [7]. S'il est vrai que cette structure intervient dans le contrôle de l'épissage, elle doit être relativement stable, et il doit donc être possible de la reconstituer à partir de deux molécules d'ARN indépendantes. Dans la deuxième approche, nous avons scindé la région de l'ARN autour de l'exon $6 \mathrm{~B}$ en deux fragments. L'un des fragments d'ARN contient 6B/IVSB7/7 et est épissé de manière très efficace. L'autre fragment porte la partie 3' terminale de l'intron IVS $A B$ riche en pyrimidines. Si l'hypothèse est correcte, le réappariement de ces deux fragments, et donc la reconstitution de la structure initiale, devrait de nouveau réprimer l'épissage de l'intron IVS B7 (figure 2B). In vitro, cet $\mathrm{ARN}$ hybride montre, en effet, une absence quasi totale d'épissage, qui va de pair avec la reconstitution de la structure originale.

En conclusion, nous avons montré que la formation d'une structure secondaire dans les cellules HeLa intervient dans le mécanisme d'exclusion de l'exon 6B. Quelles sont les bases moléculaires de cette répression ? L'épissage se fait au sein d'un complexe actif, le spliceosome. Depuis son ébauche jusqu'à sa structure finale, il subit de nombreux changements de conformation dus à des interactions successives et transitoires avec différents composants nucléaires. Dans les cellules non musculaires, cette structure pourrait masquer ou modifier l'accessibilité des sites d'épissage aux différents composants du complexe actif. Nous avons d'ores et déjà observé que l'épissage est bloqué à un stade très $m / s n^{\circ} 9$, vol. 7 , novembre 91 précoce dans l'assemblage du spliceosome, vraisemblablement avant ou juste après la fixation du snRNP U1 (le snRNA U1 semble être le premier à interagir avec le pré-messager). Nous avons également observé que l'accessibilité des bases vis-à-vis des sondes de "structure " est différente selon qu'il s'agit d'un précurseur réprimé ou non réprimé. Les différences majeures résident au niveau du site 5' d'épissage et du point de branchement de l'intron IVS B7. Là encore, on peut supposer que la répression de l'épissage de l'intron IVS B7 est due à une mauvaise interaction d'un composant nucléaire (par exemple la particule U1) avec les sites d'épissage. Cette hypothèse est testée actuellement dans le laboratoire. De nombreuses questions restent cependant à élucider. Une protéine est-elle nécessaire pour stabiliser la structure dans les cellules HeLa ? Si oui, son absence dans les cellules musculaires différenciées suffitelle pour déstabiliser la structure et permettre ainsi la reconnaissance du site 3' en amont de l'exon 6B ? A l'inverse, la reconnaissance de ce site dans les myotubes est-elle due à la présence de facteurs spécifiques?

Le repliement de structure de l'ARN est un processus largement utilisé par des systèmes aussi variés que l'atténuation de la traduction, la terminaison de la transcription, l'épissage des introns de types I et II. Il est maintenant établi que la formation de structure en tige-boucle est également impliquée dans la régulation de l'épissage alternatif des introns nucléaires où elle exerce soit un effet négatif en réprimant l'épissage, soit un effet positif en l'activant [8]

Joelle Marie ${ }^{1}$
Béatrice Clouet d'Orval ${ }^{2}$
Yves d'Aubenton-Carafa ${ }^{3}$
Pascal Sirand-Pugnet
Maria Gallego
Edward Brody
Edwecteur de recherche a l'Inserm.
(1) Directé
(2) Boursière MRT. (3) Assistant ingé-
nieur au Cnrs. ${ }^{(4)}$ Boursier MRT.
(5) Chercheur post-doctoral. (6) Directeur
de recherche au Cnrs.
Centre de génétique moléculaire, Cnrs,
91190 Gif-sur-Yvette, France.

\section{RÉFÉRENCES}

1. Smith CWJ, Patton JG, Nadal-Ginard B. Alternative splicing in the control of gene expression. Annu Rev Genet 1989; 23 : 527-77.

2. Libri D, Fiszman MY. Épissage différentiel des transcrits musculaires. médecine/sciences $1990 ; 6: 626-34$

3. Goux-Pelletan M, Libri D, d'AubentonCarafa Y, Fiszman M, Brody E, Marie J. In vitro splicing of mutually exclusive exons from the chicken $\beta$-tropomyosin gene : role of the branch point location and very long pyrimidine strech. Embo J 1990 ; 9 : 241-9.

4. Clouet d'Orval B, d'Aubenton-Carafa Y, Sirand-Pugnet P, Gallego M, Brody E, Marie J. RNA secondary structure repression of a muscle-specific exon in HeLa cell nuclear extracts. Science $1991 ; 252$ : 1823-8.

5. Libri $\mathrm{D}$, Lemonnier $M$, Meinnel $T$, Fiszman MY. A single gene codes for the $\beta$-subunit of smooth and skeletal muscle tropomyosin in the chicken. J Biol Chem $1989 ; 264$ : 2935-44.

6. Clouet d'Orval B, d'Aubenton-Carafa Y, Marie J, Brody E. Determination of an RNA structure involved in splicing inhibition of a muscle-specific exon. $J \mathrm{Mol} \mathrm{Biol}$ 1991; 221 : sous presse.

7. Libri D, Piseri A, Fiszman MY. Tissuespecific splicing in vivo of the tropomyosin gene : dependence on an RNA secondary structure. Science $1991 ; 252$ : 1842-5.

8. Chebli K, Gattoni R, Schmitt P., Hildwein $\mathrm{G}$, Stevenin J. The 216-nucleotide intron of the E1A pre-mRNA contains a hairpin structure that permits utilization of unusually distant branch acceptors. Mol Cell Biol 1989 ; 9 : 4852-61.

Remerciements

Les auteurs remercient le Ministère de la Recherche et de la Technologie pour son soutien financier.

\section{TIRÉS A PART}

J. Marie. 